\title{
Generation and characterization of tabalumab, a human monoclonal antibody that neutralizes both soluble and membrane-bound B-cell
} activating factor

\author{
This article was published in the following Dove Press journal: \\ Journal of Inflammation Research \\ 20 August 2014 \\ Number of times this article has been viewed
}

\section{Joseph Manetta \\ Holly Bina \\ Paul Ryan \\ Niles Fox \\ Derrick R Witcher \\ Kristine Kikly}

Biotechnology Discovery Research, Lilly Research Laboratories, Eli Lilly and Company, Indianapolis, IN, USA
Correspondence: Kristine Kikly Biotechnology Discovery Research, Lilly Research Laboratories, Lilly Corporate Center, Indianapolis, IN 46285, USA

$\mathrm{Tel}+\mathrm{I} 3174339605$

Fax +l 3172772934

Email kikly_kristine@lilly.com
Abstract: B-cell activating factor (BAFF) is a B-cell survival factor with a key role in B-cell homeostasis and tolerance. Dysregulated BAFF expression may contribute to autoimmune diseases or B-cell malignancies via effects on abnormal B-lymphocyte activation, proliferation, survival, and immunoglobulin secretion. Monoclonal antibodies were generated against human BAFF, characterized for species specificity and affinity, and screened for the ability to neutralize both membrane-bound and soluble BAFF. In addition, studies were undertaken to determine the relative potency of membrane-bound and soluble BAFF. Tabalumab has a high affinity for human, cynomolgus monkey, and rabbit BAFF. No binding to mouse BAFF was detected. Tabalumab was able to neutralize soluble human, cynomolgus monkey, or rabbit BAFF with equal potency. Our data demonstrate that membrane-bound BAFF can be a more potent stimulus for B-cells than soluble BAFF, and tabalumab also neutralized membrane-bound BAFF. Tabalumab prevented BAFF from binding to BAFF receptors and demonstrated pharmacodynamic effects in human BAFF transgenic mice. Tabalumab is a high-affinity human antibody with neutralizing activity against membrane-bound and soluble BAFF. Given our findings that membrane-bound BAFF can have greater in vitro potency than soluble BAFF, neutralization of both forms of BAFF is likely to be important for optimal therapeutic effect.

Keywords: autoimmunity, B-cell malignancies, B-cell survival factor, BAFF

\section{Introduction}

B-cell activating factor (BAFF) is a member of the tumor necrosis factor superfamily (TNFSF); it is also known as BLyS, THANK, TALL-1, zTNF4, and TNFSF13b. Like other members of the TNFSF, BAFF is a type II transmembrane protein that naturally forms homotrimers to make a biologically active protein. BAFF may be found in two forms: a membrane-bound form and a soluble form released from the cell surface by an unknown protease. ${ }^{1}$ Similar to other family members, an initial report suggested both forms of BAFF are biologically active, ${ }^{2}$ but it is unknown whether these two forms stimulate B-cells with equal potency.

Early literature characterizing BAFF expression indicated that it was produced predominantly by monocytes, macrophages, and dendritic cells. ${ }^{3}$ However, additional research has found that many other cell types can produce BAFF, including neutrophils, bone marrow stromal cells, follicular dendritic cells, osteoclasts, and some T-cells depending on the physiological conditions or disease state. ${ }^{4}$ 
BAFF exerts its activity by binding three different cell surface receptors. The expression of BR3 (also known as BAFF-R or TNFRSF13c) is primarily restricted to the $\mathrm{B}$-cell lineage and is first expressed on immature/transitional B-cells, continuing through subsequent B-cell developmental stages. In addition, some activated/memory subsets of T-cells express BR3. ${ }^{5}$ TACI (transmembrane activator and calcium modulator and cyclophilin ligand interactor, also known as TNFRSF13b) expression is highest on type 2 transitional (T2) and marginal zone B-cells with modest expression on follicular B-cells and undetectable levels on germinal center B-cells. ${ }^{5,6}$ BCMA (B-cell maturation antigen, also known as TNFRSF17) appears to be almost exclusively expressed on plasma cells. ${ }^{4}$ TACI and BCMA also bind a proliferation inducing ligand (APRIL), BAFF's closest homologue. B-cells may express one or more of these receptors at the same time; how the receptors influence each other's signaling is still unknown.

BAFF plays a major role in B-cell homeostasis and peripheral tolerance. Mice devoid of BAFF or BR3 are profoundly deficient in mature B-cells demonstrating that $\mathrm{BAFF}$ is required for $\mathrm{B}$-cell maturation after the transitional stage. BAFF acts as a survival factor for immature/transitional B-cells as they leave the bone marrow and enter the periphery. Using in vitro assays, BAFF has been shown to provide survival signals to prevent apoptosis of normal B-cells. ${ }^{1}$ In addition, BAFF stimulation protects malignant B-cells from apoptosis, including lymphoma, chronic lymphocytic leukemia, and multiple myeloma (MM). ${ }^{7,8}$ Importantly, BAFF stimulation rescues MM cells from dexamethasone-induced growth inhibition and cell death by signaling through both the canonical and non-canonical NF- $\kappa \mathrm{B}$ pathway ultimately upregulating anti-apoptotic genes. ${ }^{7-10}$

BAFF can also act as a co-stimulatory molecule for B-cells that have been activated through the B-cell receptor. ${ }^{4}$ Additionally, BAFF can assist B-cells as they undergo class switching to immunoglobulin $\mathrm{G}$ ( $\mathrm{IgG}$ ). Administration of exogenous BAFF to mice in conjunction with T-cellindependent or T-cell-dependent antigens can potentiate antibody responses and increase the number of plasma cells. ${ }^{1}$

Under physiologic conditions, autoreactive B-cells must compete with other B-cells for survival factors such as BAFF. Overexpression of BAFF, as seen in transgenic (Tg) mice, results in an increase in peripheral B-cells and survival of self-reactive B-cell clones that would normally have been deleted. ${ }^{11}$ Depending on the background strain, BAFF Tg mice can develop signs of autoimmune disease with age, such as autoantibodies and immune complex deposition in the kidneys. ${ }^{4}$ In addition, autoreactive B-cells have been shown to have an increased dependence on BAFF for continued survival. ${ }^{12}$

Elevated BAFF levels have been observed in patients with autoimmune diseases and B-cell malignancies. ${ }^{9,10}$ In systemic lupus erythematosus (SLE), multiple groups have demonstrated elevated serum BAFF levels, and these have been correlated with autoantibody production and disease activity. ${ }^{13,14}$ Monocytes from SLE patients have increased membrane-bound BAFF expression. ${ }^{13,15}$ In rheumatoid arthritis (RA), high BAFF levels were detected in synovial fluid (SF). ${ }^{16}$ In a study with matched serum and SF samples, BAFF levels were higher in SF suggesting a local production. $^{9}$

Therapeutic intervention in the BAFF pathway has been shown to provide clinical benefit in SLE. ${ }^{17,18}$ Belimumab, a human IgG1 antibody that binds and neutralizes BAFF, provided clinical benefit to SLE patients and is approved for the treatment of SLE. Belimumab has been shown to bind soluble BAFF with high affinity, but does not recognize membrane-bound BAFF. ${ }^{19}$ Because of the evidence suggesting a role for BAFF in the pathophysiology of multiple autoimmune diseases and B-cell malignancies, monoclonal antibodies were developed that neutralize both membranebound and soluble BAFF as a potential therapeutic approach to these diseases.

\section{Materials and methods Immunizations and hybridomas}

Monoclonal antibodies were generated using the HuMAbMouse $^{\text {TM }}$ technology at Medarex (Princeton, NJ, USA) by immunizing mice with soluble human BAFF (amino acids 133-285; Research Diagnostics Inc., Flanders, NJ, USA). Both the HCo7 and HCo12 mice were used. Mice were immunized with $15 \mu \mathrm{g}$ to $50 \mu \mathrm{g}$ soluble human BAFF in RIBI adjuvant (RIBI ImmunoChem Research, Inc, Hamilton, MT, USA), Freund's complete adjuvant or Freund's incomplete adjuvant. Eight mice producing serum antibody titers to human BAFF were injected intravenously with $10 \mu \mathrm{g}$ human BAFF in phosphate buffered saline (PBS). The spleen was harvested 3 days later from each mouse and fused with myeloma cells according to the method described by Zola. ${ }^{20}$ Hybridomas were tested for specific binding to human BAFF and to make sure they were expressing human immunoglobulin heavy and light chains by enzyme-linked immunosorbent assay (ELISA).

Microtiter plate wells were coated with either human APRIL, human BAFF, or mouse BAFF overnight at $4^{\circ} \mathrm{C}$. 
The plates were washed twice with wash buffer and blocked with casein/PBS. Tabalumab and positive control antibodies were diluted in blocking solution, plated and incubated for 1.3 hours at room temperature (RT). The plate was washed three times and then the appropriate anti-IgG (Fc specific)HRP secondary was dispensed and incubated at RT for 1 hour. The plate was washed three times and developed using an o-Phenylenediamine dihydrochloride (OPD; Sigma-Aldrich Co, St Louis, MO, USA) substrate. The substrate reaction was stopped with $1 \mathrm{~N} \mathrm{HCl}$ and the absorbance 490 was read on a Molecular Devices (Sunnyvale, CA, USA) plate reader.

Here we describe the characteristics of one of these antibodies, tabalumab (ta bal' ue mab) or LY2127399.

\section{Purification of antibodies}

Cell culture media of subcloned hybridomas was concentrated in Amicon ProFlux M12 tangential filtration systems using an Amicon S3Y30 UF membrane (EMD Millipore, Billerica, MA, USA). The concentrated media was passed over protein-A Sepharose columns (5 to $20 \mathrm{~mL}$ column) at a flow rate of $5 \mathrm{~mL} / \mathrm{min}$. The columns were washed with buffer $\mathrm{A}$ (PBS, pH 7.4) until the absorbance returned to baseline and the bound antibodies were eluted with $50 \mathrm{mM}$ citric acid, $\mathrm{pH}$ 3.2. Fractions were immediately neutralized with $1 \mathrm{MTris,}, \mathrm{pH}$ 8.0. Fractions were then analyzed by SDS-PAGE. Fractions containing antibody were pooled and concentrated using an Ultrafree centrifugal filter unit (EMD Millipore; $10 \mathrm{kDa}$ molecular weight cut-off).

\section{Surface plasmon resonance}

Antibody affinities to various species of BAFF were determined using a Biacore biosensor 2000 (GE Healthcare Bio-Sciences Corp., Piscataway, NJ, USA). Protein A was coupled via free amine groups to carboxyl groups on Flow cells 1 and 2 of a CM5 biosensor chip (GE Healthcare BioSciences Corp.). The antibody was captured on Flow cell 2, and BAFF was then injected over Flow cells 1 and 2 at increasing concentrations. Affinity for human, cynomolgus monkey, or rabbit BAFF was measured at $25^{\circ} \mathrm{C}$ using the monomeric mass of BAFF.

\section{Generation of cells expressing membrane-bound BAFF}

Membrane-bound BAFF is naturally cleaved from the membrane by one or more metalloproteases. To prevent this natural cleavage, an expression construct containing full length human BAFF was generated in which the cleavage site was mutated ( $\underline{\mathrm{KRAV}} \rightarrow \underline{\mathrm{AAAV}}){ }^{3}$ HEK293 cells were transfected with this non-cleavable BAFF construct (memBAFF) or with vector only (negative control). Abrogation of cleavage was confirmed by testing for soluble BAFF using an ELISA (R\&D Systems Kit DBLYS0) (data not shown). In order to stimulate B-cells with the transfected cells, cells were removed from the flask using enzyme free cell dissociation media (Chemicon, Bedford, MA), washed with PBS, and either fixed with $1 \%$ paraformaldehyde, or membrane preps were prepared by lysing the cells in a hypotonic solution and ultracentrifugation.

\section{BAFF quantitation assay}

A competition ELISA, using an antibody to BAFF that recognizes a conformationally-dependent epitope, was used to determine the level of BAFF in the native conformation on the memBAFF cells. Recombinant human soluble BAFF was coated on microtiter plates (Greiner Bio-One, Monroe, NC, USA), $3.6 \mathrm{ng} /$ well in PBS, overnight at $4^{\circ} \mathrm{C}$. The ELISA plate was then washed (TBS, $0.1 \%$ Tween 20) and blocked (Casein/PBS; Thermo Fisher Scientific, Waltham, MA, USA). In a polypropylene plate, serially diluted soluble BAFF, cells expressing memBAFF, or vector control cells were incubated with $4.5 \mathrm{ng}$ of anti-BAFF (clone 6B2.1) for 1 hour at RT with shaking. After the incubation, $50 \mu \mathrm{L}$ of the BAFF/antibody mixture was added to the ELISA plate and shaken for 12 minutes at RT. The plate was washed and the plate-bound anti-BAFF was developed using donkey, anti-Human IgG $(\mathrm{H}+\mathrm{L})$ HRP (Jackson ImmunoResearch Laboratories, Inc., West Grove, PA, USA) followed by OPD substrate. The plate was read on a Molecular Devices plate reader and the data analyzed using SoftMaxPro 3.1.2 software (Molecular Devices, Sunnyvale, CA, USA) using a 4 parameter curve fit. The soluble BAFF titration was used to establish the standard curve to quantify the number of memBAFF molecules on the cells.

\section{B-cell-line proliferation assay}

$\mathrm{T} 1165.17$ is a murine plasmacytoma cell line that requires low levels of interleukin (IL)-1 $\beta$ for survival, yet other cytokines can substitute for IL-1 $\beta$ in short-term proliferation assays. ${ }^{21}$ The cells were washed three times with assay media to remove IL-1 $\beta$. The BAFF activity assay was performed in Roswell Park Memorial Institute medium (RPMI)1640 supplemented with 10\% fetal bovine serum (FBS), $1 \mathrm{mM}$ sodium pyruvate, $5 \times 10^{-5} \mathrm{M} 2$-mercaptoethanol, and antibiotic/antimycotic (Gibco; Thermo Fisher Scientific, Waltham, MA, USA). BAFF in either the soluble or membrane-bound form (or an equivalent amount of vector 
control membrane) was added to a 96-well plate in triplicate. T1165.17 cells were added to each well, and the plate was incubated for 44 hours at $5 \% \mathrm{CO}_{2}, 37^{\circ} \mathrm{C}$. Cell Titer 96 viability substrate (Promega Corporation, Madison, WI, USA) was added and the plate incubated for an additional hour before reading the absorbance at 490 on a Molecular Devices plate reader. To evaluate the inhibition of BAFF by tabalumab, a sub-maximal amount of BAFF was combined with tabalumab or isotype control antibody in the 96-well plate prior to adding T1165.17 cells and then following the protocol above. The data were analyzed using a fourparameter fit and plotted as a Hill-slope curve.

\section{Primary human B-cell assay}

Primary human B-cells were isolated from peripheral blood using CD19 positive selection with the MACS magnetic isolation system (Miltenyi Biotec, Auburn, CA, USA). The B-cells were stimulated with $10 \mu \mathrm{g} / \mathrm{mL}$ anti-human $\mathrm{IgM}$ (BD Biosciences, San Jose, CA, USA, Clone G20-127; or Jackson ImmunoResearch, 109-005-043), 10 ng/mL IL-4 (R\&D Systems) and 2.3-25 ng/mL human soluble BAFF (or $5.5 \mathrm{ng} / \mathrm{mL}$ memBAFF) in the presence or absence of various concentrations of tabalumab or isotype control antibody in complete RPMI containing 10\% FBS. Three or 5 days later, ${ }^{3} \mathrm{H}$-thymidine was added and the plate incubated for 6 more hours at $37^{\circ} \mathrm{C}$ in $5 \% \mathrm{CO}_{2}$. The plate was harvested using a Filtermate cell harvester and radioactivity counted using a TopCount monitor (PerkinElmer Inc., Walther, MA, USA). Incorporation of ${ }^{3} \mathrm{H}$-thymidine was used as the measure of proliferation.

\section{BAFF binding to BAFF receptors on transfected cells}

BAFF was biotinylated using Pierce NHS-LC-biotin (Thermo Fisher Scientific); HEK293 cells were stably transfected with human BR3, human TACI, or human BCMA. Expression was confirmed with specific antibody binding and flow cytometry. The cells were removed from the cell culture flask using Enzyme Free Cell Dissociation solution (EMD Millipore), resuspended at $10^{6}$ cells $/ \mathrm{mL}$ in FACS buffer (D-PBS, $2 \% \mathrm{FBS}, 0.1 \%$ sodium azide) with $1 \mathrm{mg} / \mathrm{mL}$ goat IgG added. For cells expressing BCMA, $300 \mathrm{ng} / \mathrm{mL}$ biotinylated $\mathrm{BAFF}+50 \mu \mathrm{g} / \mathrm{mL}$ tabalumab were pre-incubated for 15 minutes at RT. For BR3, TACI, or vector control cells, $18.8 \mathrm{ng} / \mathrm{mL}$ biotinylated BAFF $+5 \mu \mathrm{g} / \mathrm{mL}$ tabalumab were pre-incubated for 15 minutes at RT. The mixture was added to cells and incubated for 20 minutes on ice. The cells were washed with FACS buffer and resuspended with
streptavidin-PE (phycoerythrin; Jackson ImmunoResearch) at a 1:150 dilution and incubated on ice for 15 minutes. The cells were washed as above and then resuspended in FACS buffer. The fluorescence was measured using a Guava EasyCyte Plus instrument (EMD Millipore) counting 2,000 cells with the Express Plus protocol. The files were converted to FCS files and the overlays were generated using WinList 5.0 (Verity Software House, Topsham, ME, USA).

\section{Human BAFF transgenic mice}

For the generation of Tg mice, a DNA fragment encoding the extracellular (soluble) region of human (hu) BAFF was cloned into a liver-specific expression vector consisting of the human Apo E gene promoter and liver enhancer (provided by John Taylor, The J David Gladstone Institutes, San Francisco, CA) modified to include a DNA fragment encoding the mouse preprotrypsin signal peptide upstream of the soluble BAFF coding region. A $6.4 \mathrm{~kb}$ DNA fragment encompassing the Apo E promoter-huBAFF fusion gene was isolated and micro-injected into the pronuclei of $\mathrm{FVB} / \mathrm{N}$ strain zygotes as previously described..$^{22}$

The presence of the transgene in newborn founder mice and progeny was confirmed by polymerase chain reaction (PCR) analysis of tail or toe tissue using primers specific for the human ApoE sequences. The overexpression of soluble human BAFF in the circulation was confirmed by ELISA (R\&D Systems Kit DBLYS0).

\section{Cell culture and flow cytometry}

Normal human monocytes were isolated by CD14 microbead separation from peripheral blood mononuclear cells (PBMCs). PBMCs were isolated from heparinized blood using Ficoll-Paque PLUS (GE Healthcare Bio-Sciences Corp). Positive selection of the monocyte population was performed by magnetic cell sorting (MACS, Miltenyi Biotec) using CD14 microbeads, according to the manufacturer's protocol. Normal human monocytes $\left(1 \times 10^{6}\right.$ cells $\left./ \mathrm{mL}\right)$ were cultured in RPMI1640 supplemented with $10 \%$ fetal calf serum, $100 \mathrm{U} / \mathrm{mL}$ penicillin, and $100 \mu \mathrm{g} / \mathrm{mL}$ streptomycin. Cells were maintained at $5 \% \mathrm{CO}_{2}$ at $37^{\circ} \mathrm{C}$. Cells were treated with $1 \mu \mathrm{g} / \mathrm{mL}$ of INF $\gamma$ (R\&D Systems) or media alone for 3 days. Cells were stained with the following antibodies: antiCD14-PE (eBioscience, San Diego, CA, USA), tabalumabAlexa 488, or isotype control-Alexa 488 (labeled in-house using Alexa488 kit from Thermo Fisher Scientific). Flow cytometric analysis was carried out using FC500 flow cytometer (Beckman Coulter, Indianapolis, IN, USA) and FlowJo software (Tree Star, Ashland, OR, USA). 
Splenocytes from human BAFF Tg mice were pelleted and resuspended in cold FACS buffer (Hank's Balanced Salt Solution [HBSS; Hyclone SH30268.02] containing $2 \%$ FBS [Thermo Fisher Scientific] and $0.1 \%$ sodium azide) at a density of $3 \times 10^{7}$ cells $/ \mathrm{mL}$. A $20 \mu \mathrm{L}$ aliquot of each splenocyte sample was transferred into a 96-well plate and $10 \mu \mathrm{L}$ of rat anti-mouse CD45R/B220-Cychrome (0553091; BD Biosciences) in FACS buffer were added to the appropriate wells for a final concentration of 1:200. The plates were sealed and incubated at $4^{\circ} \mathrm{C}$ for 30 minutes. The stained cells were washed with cold FACS buffer, pelleted, and resuspended in $300 \mu \mathrm{L}$ cold FACS buffer. Data were immediately acquired on a Beckman Coulter FC500 flow cytometer and analyzed using CXP Analysis and Excel software.

\section{Neutralization of human BAFF in transgenic mice}

Mice were treated (Day 0) with either human IgG4 isotype control or tabalumab, $500 \mu \mathrm{g}$ in $200 \mu \mathrm{L}$ PBS delivered subcutaneously. Animals were taken off study 2, 4, 8, 11, 16 and 23 days after administration of antibody. Splenocytes were processed into a single cell suspension into cold HBSS (14025; Thermo Fisher Scientific), and then put through a $70 \mu \mathrm{m}$ filter. The cells were pelleted by centrifugation and resuspended in cold PharmLyse (BD Biosciences) for 3 minutes. Ten $\mathrm{mL}$ cold HBSS was added to dilute the lysing buffer, and the samples were spun to pellet the cells. The cells were resuspended with $10 \mathrm{~mL}$ cold HBSS and counted. ViaCount Reagent (4000-0040; EMD Millipore) was used to determine absolute cell counts and viability of the splenocyte samples using the Guava EasyCyte Plus flow cytometer (EMD Millipore) and analyzed with CytoSoft 5.2 (EMD Millipore) and Excel software.

\section{p52 binding assay}

Plates coated with p52 binding DNA (Clontech S3528; Clontech Laboratories, Mountain View, CA, USA) were blocked for at least 15 minutes with 3\% blocking solution (Clontech S2794) made in 1× Transfactor buffer (Clontech S2778). Splenocyte lysates from human BAFF Tg mice were diluted to $100 \mu \mathrm{g} / \mathrm{mL}$ final protein concentration with the blocking solution. The blocking solution was removed from the plate, and $50 \mu \mathrm{L}$ of diluted sample (run in duplicate) was added to the plate and incubated at RT for 1 hour. The plate was washed three times with blocking solution. One hundred $\mu \mathrm{L}$ of a 1:1000 dilution of rabbit anti-p52 (Upstate, Lake Placid, NY, USA) was added to each well and incubated at RT for 1 hour. The plate was washed as before and $100 \mu \mathrm{L}$ of goat anti-rabbit IgG - HRP (Jackson ImmunoResearch) at a 1:2000 dilution was added to each well. The plate was incubated for 40 minutes at RT. The plate was washed three times with $1 \times$ Transfactor buffer and then 1 time with PBS. One hundred $\mu \mathrm{L}$ of substrate (Thermo Fisher Scientific) was added and the plate read on a PerkinElmer Victor3 (PerkinElmer Inc.) reader using the Wallac 1420 luminescence software (PerkinElmer Inc.).

\section{Results}

\section{Binding and affinity of tabalumab}

Tabalumab was obtained by immunization of human immunoglobulin Tg mice with soluble human BAFF resulting in many hybridomas of multiple isotypes. Initially, hybridomas were tested for binding to human BAFF and to make sure they were expressing human immunoglobulin heavy and light chains. There was no binding to APRIL, the family member with the highest homology to BAFF. Affinity measurements using surface plasmon resonance indicated that tabalumab had a high affinity for human $(126 \mathrm{pM} \pm 37$ standard deviation [SD]), cynomolgus monkey (101 pM $\pm 78 \mathrm{SD}$ ), and rabbit (81 $\mathrm{pM} \pm 24 \mathrm{SD}$ ) soluble BAFF. Binding to mouse BAFF was not detected by ELISA (data not shown).

The hybridoma producing the antibody that eventually became tabalumab was a human $\operatorname{IgG1}$ isotype. Since our goal was complete neutralization of BAFF without depletion of BAFF-expressing cells, we engineered the isotype to human IgG4. This should reduce interactions with Fc receptors and eliminate antibody-dependent cellular cytotoxicity and complement-dependent cytotoxicity. It is known that human IgG4 can form two half antibodies (halfmers) each with a single heavy and light chain and these can recombine in vivo. ${ }^{23}$ To prevent half-mer formation, a specific serine-to-proline mutation has been engineered into the hinge region of human $\mathrm{IgG} 4$ connecting the $\mathrm{CH} 1$ and $\mathrm{CH} 2$ of the heavy chain. This specific mutation makes the hinge more similar to that of the human IgG1 subclass, and has been shown to minimize the antibody-molecule heterogeneity. ${ }^{23}$

Tabalumab was able to bind to a HEK293 cell line stably expressing non-cleavable human BAFF, or membrane-bound BAFF (Figure 1A). To confirm binding to cells naturally expressing membrane-bound BAFF, human monocytes were activated with IFN- $\gamma$, which is known to induce BAFF expression. ${ }^{24}$ Figure 1B shows that tabalumab binds membranebound BAFF on activated human monocytes. 
A

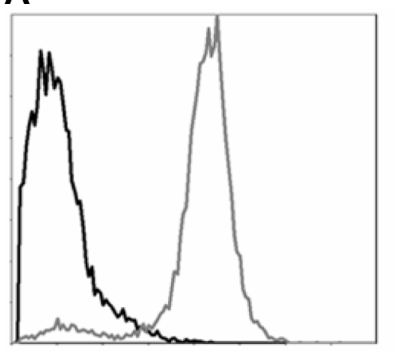

293/BAFF
B

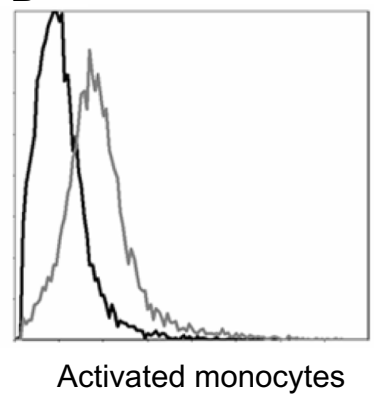

Figure I Tabalumab binds membrane-bound BAFF on transfected cells or activated human monocytes.

Notes: (A) HEK293 cells were stably transfected with non-cleavable human BAFF. Membrane-bound BAFF was detected with Alexa 488-labeled tabalumab (gray histogram) compared with Alexa 488-labeled isotype control (black histogram). (B) Purified human CDI4+ monocytes were activated with IFN- $\gamma$ for 3 days. Membrane-bound BAFF was detected with Alexa 488-labeled tabalumab (gray histogram) compared with Alexa 488-labeled isotype control (black histogram). Data are from one experiment but representative of $n=12$ experiments $(A)$ or ten donors (B).

Abbreviation: BAFF, B-cell activating factor.

\section{Biological activity of BAFF}

Similar to other TNFSF members, both the soluble and membrane-bound form of BAFF could be biologically active. To confirm this in vitro, a B-cell line or primary human B-cells were stimulated with either soluble human BAFF or cells expressing a non-cleavable form of membrane-bound BAFF. Data in Figure 2A show that membrane-bound BAFF is a more potent stimulus of 1165.17 cells, a murine plasmacytoma cell line, compared with soluble BAFF. In contrast, primary human B-cells responded slightly better to soluble BAFF (Figure 2B). The differences in response could be due to species' differences or in receptor expression. T1165.17 cells strongly express TACI whereas primary human B-cells predominantly express BR3. The results demonstrate that soluble and membrane-bound BAFF were found to be biologically active.

\section{Tabalumab neutralizes soluble and membrane-bound BAFF}

Neutralization of BAFF was tested using T1165.17 cells and primary human B-cells. Using T1165.17 cells, the $\mathrm{IC}_{50}$ (half maximal inhibitory concentration) for tabalumab neutralization of soluble human, cynomolgus monkey, or rabbit BAFF was $104 \mathrm{pM}, 143 \mathrm{pM}$, and $176 \mathrm{pM}$, respectively (Figure 3). Tabalumab was tested for the ability to inhibit human B-cell proliferation induced by soluble or membrane-bound human BAFF in the presence of IL-4 and anti-human IgM. Figure 4A illustrates the ability of a dose titration of tabalumab to neutralize the effect of soluble BAFF on anti-IgM/IL-4-induced B-cell proliferation. Figure 4B demonstrates the ability of tabalumab to neutralize the membrane-bound form of BAFF compared with the soluble form of BAFF. Neutralization of cynomolgus monkey membranebound BAFF was also observed (data not shown).

\section{Tabalumab inhibits BAFF binding to $\mathrm{BR} 3, \mathrm{TACl}$, and $\mathrm{BCMA}$}

The ability of tabalumab to interfere with BAFF binding to each of the three different receptors for BAFF was investigated. HEK293 cells were transfected with either BR3, TACI, or BCMA, then incubated with BAFF in the presence of tabalumab or isotype control. As shown in Figure 5, tabalumab prevents the binding of BAFF to BR3, TACI, or BCMA on the cell surface. In contrast, once BAFF has docked into a receptor, tabalumab is unable to recognize it (data not shown). Tabalumab neutralizes the biological
A

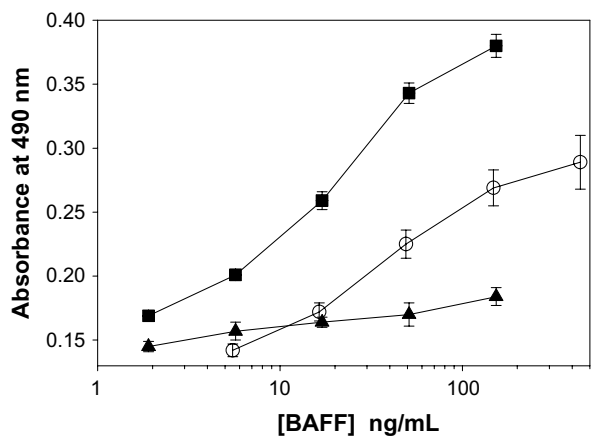

B

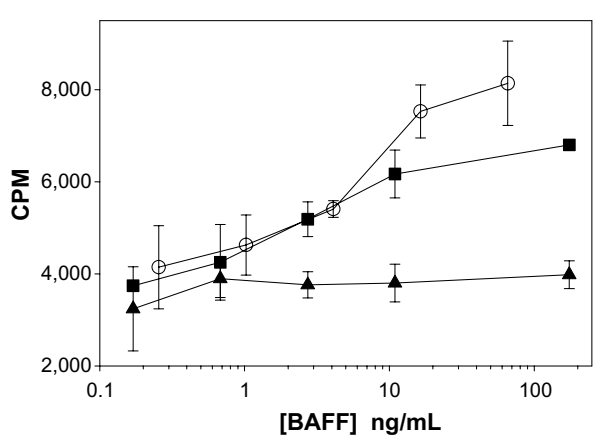

Figure 2 B-cells proliferate in response to soluble or membrane-bound BAFF.

Notes: $($ A ) TII 65.17 cells were stimulated with a dose titration of membrane-bound BAFF $(\boldsymbol{\square})$, soluble BAFF $(\bigcirc)$, or vector control cells $(\bullet)$. Proliferation was measured using a colorimetric method for determining the number of viable cells at 44 hours. (B) Human CDI9+ B-cells were co-stimulated with a dose titration of membrane-bound $\operatorname{BAFF}(\boldsymbol{\square})$, soluble BAFF $(\bigcirc)$, or vector control cells $(\boldsymbol{\Delta})$ in the presence of anti-lgM and IL-4. Proliferation was measured using ${ }^{3} \mathrm{H}$-thymidine incorporation at 72 hours. Data are presented as mean \pm SD.

Abbreviations: BAFF, B-cell activating factor; CPM, counts per minute; Ig, immunoglobulin; IL, interleukin; SD, standard deviation. 


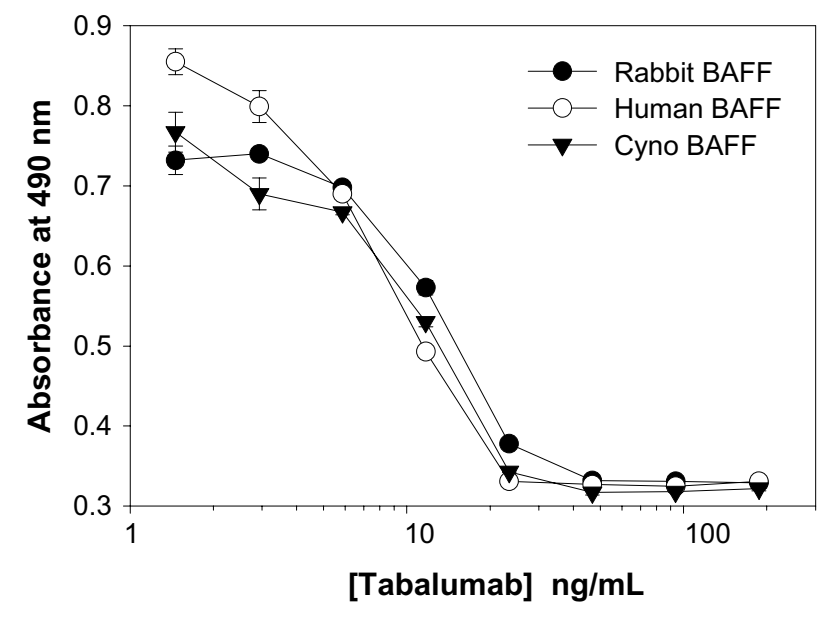

Figure 3 Tabalumab neutralization of soluble BAFF from multiple species. Notes: IC $C_{50}$ was calculated for soluble human BAFF (IC $: 104$ pM, 95\% CI 96-I I 2 pM), soluble cynomolgus monkey BAFF (IC I0 $_{50}$ I 43 pM, 95\% Cl I26-162 pM), or soluble rabbit BAFF (IC ${ }_{50}$ I 176 pM, 95\% Cl I70-182 pM).TII 65.17 cells were stimulated with 365 pM BAFF and proliferation was measured using a colorimetric method for determining the number of viable cells at 44 hours. Data are presented as mean \pm SD.

Abbreviations: BAFF, B-cell activating factor; $\mathrm{Cl}$, confidence interval; cyno, cynomolgus monkey; $I C_{50}$, half maximal inhibitory concentration; SD, standard deviation.

activity of BAFF due to the ability to prevent binding of BAFF to its receptors.

\section{Pharmacodynamic response of tabalumab in human BAFF transgenic mice}

In order to determine the ability of tabalumab to neutralize BAFF in vivo, human BAFF Tg mice were used. Characterization of the BAFF Tg mice included demonstration of human BAFF in serum samples (data not shown). Untreated non-Tg mice had an average splenocyte count of $81 \times 10^{6}$ cells while untreated BAFF Tg mice had an average splenocyte count of $156 \times 10^{6}$ cells. Human BAFF
Tg mice had 2.5-fold more splenic B-cells compared with non- $T$ g mice, $118 \times 10^{6}$ versus $48 \times 10^{6}$, respectively (Figure 6A). BR3 signals through the non-canonical $\mathrm{NF}-\kappa \mathrm{B}$ pathway resulting in the processing of p100 to p52. ${ }^{25}$ Human BAFF Tg mice had an increased level of p52 in splenocyte lysates when compared with non- $\mathrm{Tg}$ mice as determined by both $\mathrm{p} 52$ binding assay (Figure 6B), and Western blot analysis (data not shown) reflecting increased BR3 signaling.

Mice were treated with a single dose of tabalumab or an isotype control antibody, and splenic B-cells and p52 levels were monitored for 23 days. Tabalumab is specific for human BAFF and will not inhibit the activity of the endogenous mouse BAFF. The splenic B-cell count significantly decreased between Days 4 and 8 following administration of tabalumab and remained near the non-Tg level until Day 16 (Figure 6A). B-cell numbers returned to baseline BAFF Tg levels by Day 23.

The p52 binding assay data show that tabalumab-treated mice consistently had lower $\mathrm{p} 52$ levels than isotype controltreated animals beginning at Day 2, and the levels remained reduced through Day 16 (Figure 6B). The p52 levels returned to near the BAFF Tg level at Day 23. In addition, Western blot data show that the p100 levels increased in the lysates as the $\mathrm{p} 52$ levels decreased, indicating that the processing of p100 to p52 was retarded following treatment with tabalumab (Figure 6C). The in vivo neutralization of human BAFF using tabalumab in human BAFF Tg mice resulted in a decrease in signaling through the non-canonical NF- $\kappa B$ pathway and a reduction in B-cells in the spleen. The reduction in $\mathrm{p} 52$ levels in the spleen preceded the B-cell reduction by several days.

B

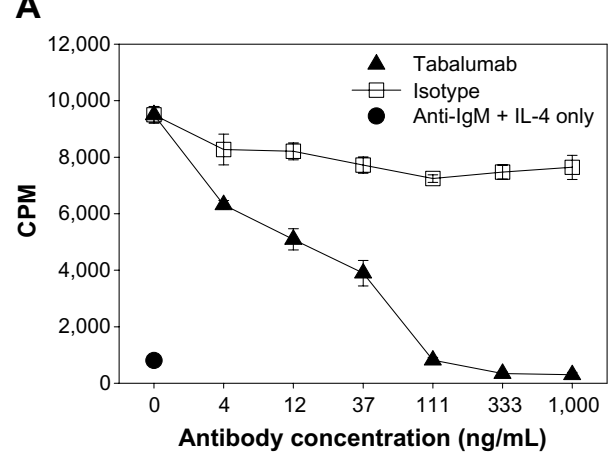

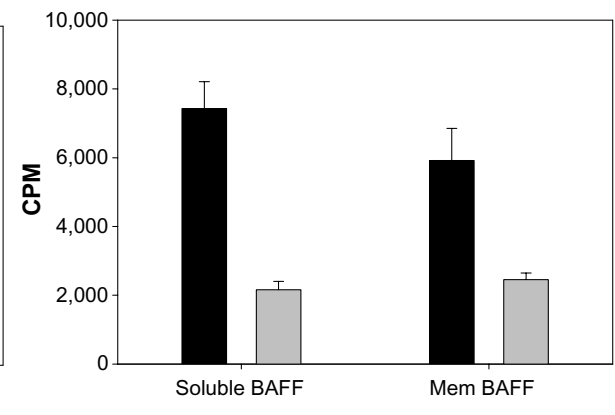

Figure 4 Tabalumab neutralizes soluble or membrane-bound human BAFF co-stimulation of B-cells.

Notes: (A) Primary human CDI9+ B-cells were co-stimulated with $25 \mathrm{ng} / \mathrm{mL}$ soluble BAFF plus anti-lgM/IL-4 in the presence of a dose response of tabalumab or isotype control. Proliferation was measured using ${ }^{3} \mathrm{H}$-thymidine incorporation at day 5. (B) Primary human CDI9+ B-cells were co-stimulated with $2.3 \mathrm{ng} / \mathrm{mL}$ soluble BAFF or $5.5 \mathrm{ng} / \mathrm{mL}$ membrane-bound BAFF plus anti-lgM/L-4. Gray bars represent cells treated with I $\mu \mathrm{g} / \mathrm{mL}$ tabalumab, black bars represent cells treated with I $\mu \mathrm{g} / \mathrm{mL}$ isotype control. Stimulation with anti-lgM/IL-4 alone produced a relatively low proliferative signal of $3640 \pm 592 \mathrm{CPM}$. Proliferation was measured using ${ }^{3} \mathrm{H}$-thymidine incorporation at day 3. Data are presented as mean \pm SD.

Abbreviations: BAFF, B-cell activating factor; CPM, counts per minute; Ig, immunoglobulin; IL, interleukin; mem, membrane-bound; SD, standard deviation. 

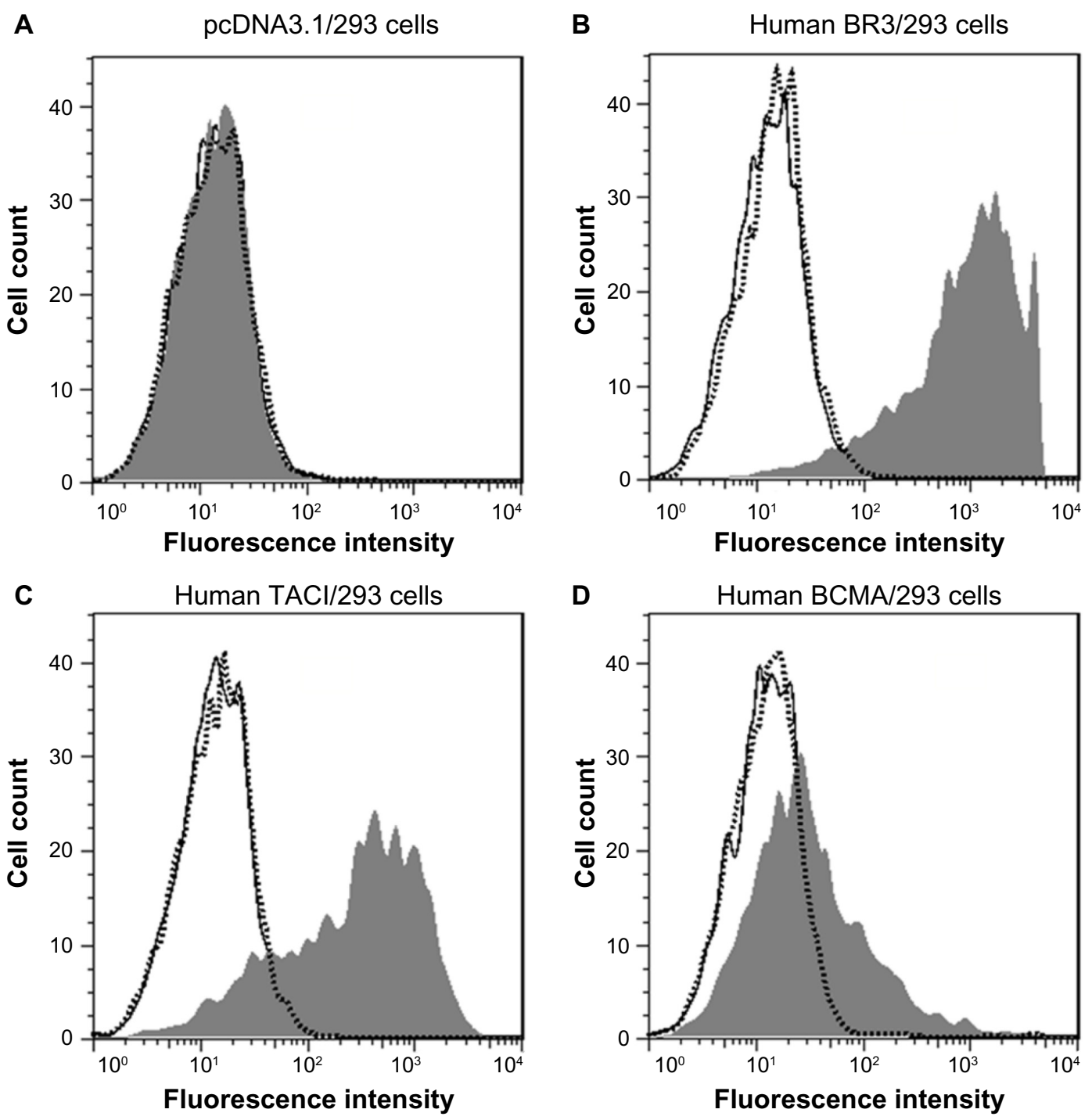

Figure 5 Tabalumab prevents BAFF binding to the three BAFF receptors.

Notes: HEK293 cells were transfected with either empty vector (A), human BR3 (B), human TACl (C), or human BCMA (D). Tabalumab or isotype control was preincubated with soluble biotinylated BAFF for 15 minutes prior to addition to each cell line. BAFF binding is visualized by addition of streptavidin-PE and the fluorescence is read on a flow cytometer. Black solid line represents cells incubated with buffer only; dark gray histogram represents cells and isotype control antibody + biotinylated BAFF; dotted line with open histogram represents cells and tabalumab + biotinylated BAFF.

Abbreviation: BAFF, B-cell activating factor; PE, phycoerythrin.

\section{Discussion}

BAFF plays a critical role in the development and maintenance of normal B-cell populations and in numerous B-cell functions. Several lines of evidence also suggest that BAFF contributes to pathophysiology in multiple autoimmune diseases and B-cell malignancies. ${ }^{4}$

Similar to TNF, both soluble and membrane-bound BAFF are biologically active, but it is not clear whether there are distinct roles for membrane-bound BAFF compared with soluble BAFF. Despite years of research and several unique pharmacologic agents for TNF neutralization, the distinct roles for each form of TNF still remain elusive. ${ }^{26,27}$ Our data show that depending on the context, membrane-bound BAFF can be a more potent stimulus than cleaved soluble BAFF. This is consistent with a report by Bossen et $\mathrm{a}^{28}$ demonstrating that membrane-bound BAFF is at least 50-fold more active on cells expressing BCMA. In addition, we have confirmed reports that T-cells can be co-stimulated with BAFF through BR3, but only if the BAFF is immobilized rather than soluble. ${ }^{5}$ This suggests that T-cells require membranebound BAFF expressed by an antigen-presenting cell for co-stimulation. The fact that membrane-bound BAFF is tethered to a cell suggests that it will play a role in direct cell-to-cell communication.

The majority of soluble BAFF appears to be produced by radiation-resistant stromal cells, as lethally irradiated mice 


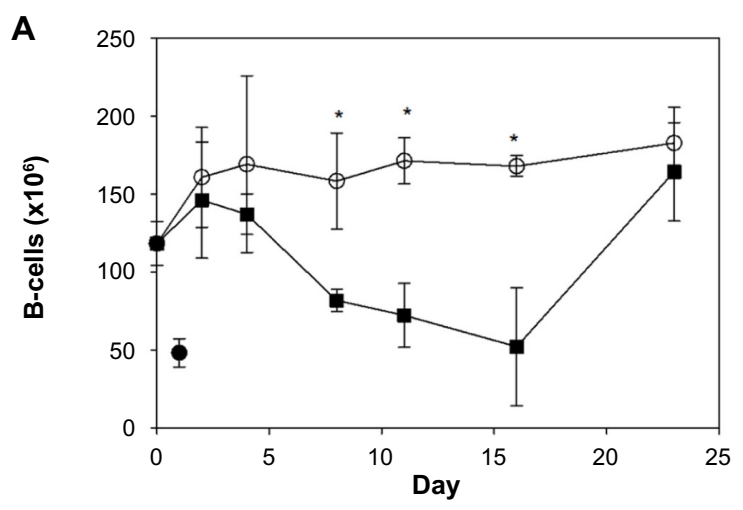

B

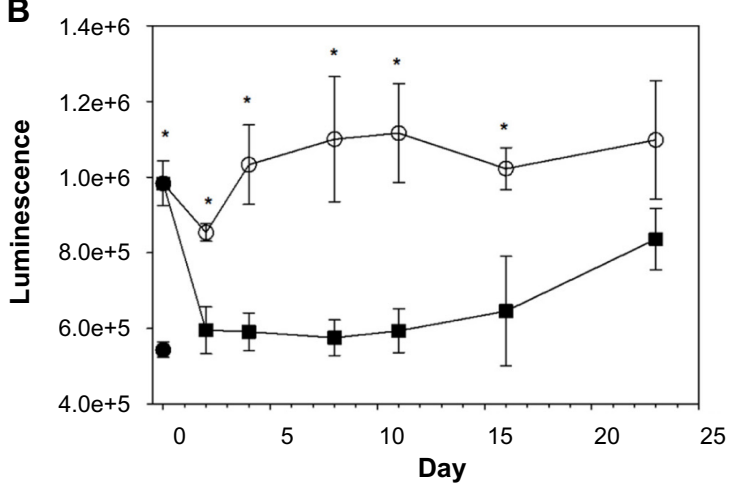

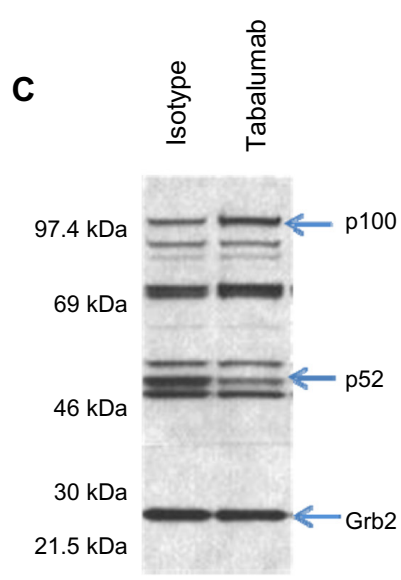

Day 4
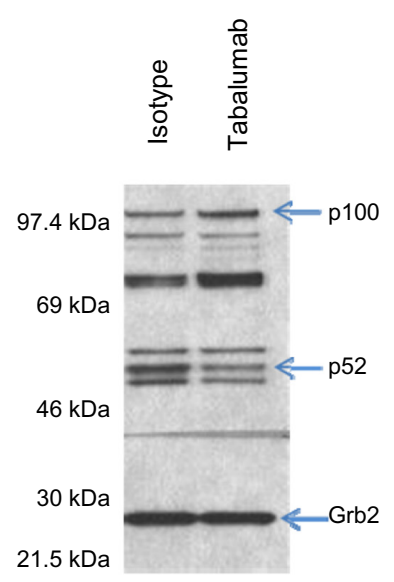

Day 8
Figure 6 Administration of tabalumab to human BAFF transgenic mice leads to decreased $\mathrm{B}$-cells and a reduction in non-canonical NF- $\mathrm{KB}$ signaling.

Notes: Tabalumab $(\boldsymbol{\square})$ or isotype control $(\bigcirc)$ was injected SC on day 0. Mice were sacrificed on days 2, 4, 8, II, I6, and 23. (A) Splenic B-cells were enumerated by flow cytometry using B220; (B) p52 levels in the spleen were evaluated by a binding assay; (C) Western blot showing bands for p 100 and p52 in splenocyte lysates; Grb2 was monitored to ensure equivalent loading of wells. * represents $P$-value $<0.05$. $(\bullet)$ represents an untreated non-transgenic mouse.

Abbreviations: BAFF, B-cell activating factor; SC, subcutaneously.

reconstituted with bone marrow from BAFF-deficient mice have normal levels of serum-soluble BAFF. ${ }^{29}$ Soluble BAFF appears to be sufficient for normal B-cell maturation. BAFF-deficient mice administered exogenous soluble BAFF can restore normal B-cell development and T-independent antibody response. ${ }^{30}$
Recent data suggest that local BAFF production by $\mathrm{T}$ follicular helper $\left(\mathrm{T}_{\mathrm{FH}}\right)$ cells is required to promote and maintain germinal center B-cells that achieve high affinity B-cell receptor maturation. ${ }^{6}$ The data suggested that $\mathrm{T}_{\mathrm{FH}}$ cells express membrane-bound BAFF locally in the germinal center, although cleavage from the cell surface to produce soluble BAFF in the microenvironment cannot be ruled out.

An attempt to create mice expressing only membranebound BAFF was reported by Bossen et a ${ }^{30}$ using a transgene containing full-length mouse BAFF with two mutations to prevent cleavage, overexpressed in BAFF-deficient mice. The resulting phenotype was similar to BAFF-knockout mice, and they concluded that membrane-bound BAFF was unable to support normal B-cell development. Unfortunately, the authors were unable to demonstrate the expression of membrane-bound BAFF on any cells or tissues from these mice, a key piece of evidence required for proper interpretation of the data.

Membrane-bound BAFF expressed by fibroblast-like synoviocytes cultured from patients with RA appears to be critical for the local activation of B-cells resulting in re-expression of enzymes involved in immunoglobulin gene rearrangement, RAG1 and RAG2. ${ }^{31}$ The effect could be inhibited in vitro with anti-BAFF or shRNA gene interference to downregulate membrane-bound BAFF expression, however, soluble BAFF could not recapitulate B-cell induction of RAG expression. The reactivation of B-cells in the synovium, outside the context of a normal germinal center, may lead to loss of self-tolerance and further promote autoreactivity.

The most reproducible cell type found to express membrane-bound BAFF is the activated monocyte. We observed good staining of activated monocytes either using tabalumab as an intact antibody or a Fab fragment of tabalumab, precluding the possibility for Fc binding (unpublished observation). Interestingly, we were unable to see binding to resting monocytes in culture or in whole blood from healthy volunteers. Despite a few reports that B-cells express $\mathrm{BAFF},{ }^{32,33}$ we were not able to observe tabalumab binding to B-cells in whole blood from healthy donors, even at concentrations up to $100 \mu \mathrm{g} / \mathrm{mL}$ (unpublished observation). This is consistent with our data that B-cells do not express BAFF mRNA, even following activation, and that once BAFF is docked into a receptor on a B-cell, tabalumab is unable to recognize BAFF.

Because tabalumab does not recognize mouse BAFF, it is not feasible to evaluate tabalumab in preclinical disease models. Tg mice expressing soluble human BAFF were used 
to confirm in vivo neutralization of BAFF by tabalumab demonstrating a reduction in $\mathrm{NF}-\kappa \mathrm{B}$ signaling and a reduction in B-cells in the spleen. The increased splenocyte number in the BAFF Tg mice was a result of increased numbers of B-cells due to the proliferative/survival effect of human BAFF on murine B-cells, as demonstrated by our studies with tabalumab. The only preclinical disease model that tabalumab was tested in was an MM xenograft model that also included a human bone graft as a source of human BAFF. ${ }^{10}$ Treatment of the mice with tabalumab demonstrated a significant reduction in tumor burden, prolongation of survival, a decrease in osteoclast recruitment and activation resulting in less lytic lesions in the bone. ${ }^{10}$

Several different BAFF antagonists with distinct mechanisms of action, and therefore potentially differing effects on patient outcomes, are being investigated in clinical trials. Belimumab is a human IgG1 monoclonal antibody that neutralizes only soluble BAFF, and it was recently approved for the treatment of SLE. ${ }^{17-19}$ The rationale for selecting an antibody to neutralize only soluble BAFF is unclear in light of the data showing expression on activated monocytes, ${ }^{24}$ upregulation on monocytes from SLE patients, ${ }^{13,15}$ and in vitro biological activity. ${ }^{2}$ In contrast, tabalumab is a human IgG4-variant monoclonal antibody that neutralizes both soluble and membrane-bound BAFF. Using a binding assay for $\mathrm{Fc}$ receptors and $\mathrm{C} 1 \mathrm{q}$, tabalumab does not bind to any of the Fc $\gamma$ receptors tested (CD16a, CD32a, and CD64) nor to the complement component $\mathrm{C} 1 \mathrm{q}$ (data not shown). These results suggest that it is highly unlikely that tabalumab will be able to induce Fc-mediated biological effects in vivo, an important consideration since our goal was complete neutralization of BAFF without depletion of BAFF-expressing cells.

Tabalumab has demonstrated the expected pharmacokinetic and pharmacodynamic responses in human clinical trials based on preclinical studies in cynomolgus monkeys. ${ }^{34}$ At doses ranging from $0.01 \mathrm{mg} / \mathrm{kg}$ to $8.0 \mathrm{mg} / \mathrm{kg}$, a single intravenous dose of tabalumab exhibited nonlinear pharmacokinetics suggesting target-mediated clearance at low doses due to binding of membrane-bound $B A F F .{ }^{35}$ In patients with RA treated with multiple infusions of tabalumab, there was a transient increase in circulating B-cells followed by a reduction. The most sensitive population measured was naïve B-cells $\left(\mathrm{CD} 20+\mathrm{IgD}+\mathrm{CD} 27^{-}\right)$, with an approximate $60 \%$ reduction in circulating B-cells over the 24 week study. ${ }^{35}$ Curiously, there was an increase in circulating memory B-cells (CD20 + IgD-CD27+) for at least 16 weeks, however, they returned to baseline by the end of the study. The mechanism responsible for the increase in circulating memory B-cells is unknown but may be related to changes in homing ability of the cells. ${ }^{36}$ These pharmacodynamic observations are consistent with other BAFF antagonists in clinical development, ${ }^{37,38}$ however, the clinical relevance is unknown.

\section{Conclusion}

Both membrane-bound BAFF and soluble BAFF are biologically active, suggesting that a greater clinical response might be achieved by inhibiting both forms of BAFF instead of either BAFF form alone. However, to date it has been difficult to determine if the different BAFF forms have unique, non-overlapping biological effects, so this hypothesis remains speculative. Tabalumab is a human IgG4 antibody that neutralizes both soluble and membrane-bound BAFF. We are awaiting the outcome of several clinical trials, evaluating the efficacy of tabalumab for treatment of autoimmune disease and MM.

\section{Acknowledgments}

The authors would like to acknowledge Tonya Pohl for screening the hybridomas, Anna Bailey for assistance with the in vivo studies, and Robert Benschop and Matt Linnik for critical reading of the manuscript.

\section{Disclosure}

The authors report no conflicts of interest in this work.

\section{References}

1. Mackay F, Browning JL. BAFF: a fundamental survival factor for B cells. Nat Rev Immunol. 2002;2(7):465-475.

2. Schneider P, MacKay F, Steiner V, et al. BAFF, a novel ligand of the tumor necrosis factor family, stimulates B cell growth. J Exp Med. 1999;189(11):1747-1756.

3. Nardelli B, Belvedere O, Roschke V, et al. Synthesis and release of B-lymphocyte stimulator from myeloid cells. Blood. 2001;97(1): 198-204.

4. Mackay F, Schneider P. Cracking the BAFF code. Nat Rev Immunol. 2009;9(7):491-502.

5. Ng LG, Sutherland AP, Newton R, et al. B cell-activating factor belonging to the TNF family (BAFF)-R is the principal BAFF receptor facilitating BAFF costimulation of circulating T and B cells. J Immunol. 2004;173(2):807-817.

6. Goenka R, Matthews AH, Zhang B, et al. Local BLyS production by $\mathrm{T}$ follicular cells mediates retention of high affinity $\mathrm{B}$ cells during affinity maturation. J Exp Med. 2014;211(1):45-56.

7. Moreaux J, Legouffe E, Jourdan E, et al. BAFF and APRIL protect myeloma cells from apoptosis induced by interleukin 6 deprivation and dexamethasone. Blood. 2004;103(8):3148-3157.

8. Haiat S, Billard C, Quiney C, Ajchenbaum-Cymbalista F, Kolb JP. Role of BAFF and APRIL in human B-cell chronic lymphocytic leukaemia. Immunology. 2006;118(3):281-292.

9. Cheema GS, Roschke V, Hilbert DM, Stohl W. Elevated serum B lymphocyte stimulator levels in patients with systemic immune-based rheumatic diseases. Arthritis Rheum. 2001;44(6):1313-1319. 
10. Neri P, Kumar S, Fulciniti MT, et al. Neutralizing B-cell activating factor antibody improves survival and inhibits osteoclastogenesis in a severe combined immunodeficient human multiple myeloma model. Clin Cancer Res. 2007;13(19):5903-5909.

11. Thien M, Phan TG, Gardam S, et al. Excess BAFF rescues self-reactive $\mathrm{B}$ cells from peripheral deletion and allows them to enter forbidden follicular and marginal zone niches. Immunity. 2004;20(6):785-798.

12. Lesley R, Xu Y, Kalled SL, et al. Reduced competitiveness of autoantigen-engaged $\mathrm{B}$ cells due to increased dependence on BAFF. Immunity. 2004;20(4):441-453.

13. Stohl W, Metyas S, Tan SM, et al. B lymphocyte stimulator overexpression in patients with systemic lupus erythematosus: longitudinal observations. Arthritis Rheum. 2003;48(12): 3475-3486.

14. Petri M, Stohl W, Chatham W, et al. Association of plasma B lymphocyte stimulator levels and disease activity in systemic lupus erythematosus. Arthritis Rheum. 2008;58(8):2453-2459.

15. Harigai M, Kawamoto M, Hara M, Kubota T, Kamatani N, Miyasaka N Excessive production of IFN-gamma in patients with systemic lupus erythematosus and its contribution to induction of B lymphocyte stimulator/B cell-activating factor/TNF ligand superfamily-13B. J Immunol. 2008;181(3):2211-2219.

16. Tan SM, Xu D, Roschke V, et al. Local production of B lymphocyte stimulator protein and APRIL in arthritic joints of patients with inflammatory arthritis. Arthritis Rheum. 2003;48(4):982-992.

17. Navarra SV, Guzman RM, Gallacher AE, et al. Efficacy and safety of belimumab in patients with active systemic lupus erythematosus: a randomised, placebo-controlled, phase 3 trial. Lancet. 2011;377(9767) 721-731

18. Furie R, Petri M, Zamani O, et al. A phase III, randomized, placebocontrolled study of belimumab, a monoclonal antibody that inhibits B lymphocyte stimulator, in patients with systemic lupus erythematosus. Arthritis Rheum. 2011;63(12):3918-3930.

19. Baker KP, Edwards BM, Main SH, et al. Generation and characterization of LymphoStat-B, a human monoclonal antibody that antagonizes the bioactivities of B lymphocyte stimulator. Arthritis Rheum. 2003;48(11):3253-3265.

20. Zola H. Monoclonal Antibodies: A Manual of Techniques. Boca Raton (FL): CRC Press; 1987.

21. Tosato G, Seamon KB, Goldman ND, et al. Monocyte-derived human B-cell growth factor identified as interferon-beta 2 (BSF-2, IL-6). Science. 1988;239(4839):502-504.

22. Fox N, Solter D. Expression and regulation of the pituitary- and placenta-specific human glycoprotein hormone alpha-subunit gene is restricted to the pituitary in transgenic mice. Mol Cell Biol. 1988;8(12): $5470-5476$.

23. Labrijn AF, Buijsse AO, van den Bremer ET, et al. Therapeutic IgG4 antibodies engage in Fab-arm exchange with endogenous human IgG4 in vivo. Nat Biotechnol. 2009;27(8):767-771.

24. Moore PA, Belvedere O, Orr A, et al. BLyS: member of the tumor necrosis factor family and B lymphocyte stimulator. Science. 1999;285(5425):260-263.
25. Claudio E, Brown K, Park S, Wang H, Siebenlist U. BAFF-induced NEMO-independent processing of NF-kappa B2 in maturing B cells. Nat Immunol. 2002;3(10):958-965.

26. Grell M, Douni E, Wajant H, et al. The transmembrane form of tumor necrosis factor is the prime activating ligand of the $80 \mathrm{kDa}$ tumor necrosis factor receptor. Cell. 1995;83(5):793-802.

27. Horiuchi T, Mitoma H, Harashima S, Tsukamoto H, Shimoda T. Transmembrane TNF-alpha: structure, function and interaction with anti-TNF agents. Rheumatology (Oxford). 2010;49(7):1215-1228.

28. Bossen C, Cachero TG, Tardivel A, et al. TACI, unlike BAFF-R, is solely activated by oligomeric BAFF and APRIL to support survival of activated B cells and plasmablasts. Blood. 2008;111(3):1004-1012.

29. Gorelik L, Gilbride K, Dobles M, Kalled SL, Zandman D, Scott ML. Normal B cell homeostasis requires B cell activation factor production by radiation-resistant cells. J Exp Med. 2003;198(6):937-945.

30. Bossen C, Tardivel A, Willen L, et al. Mutation of the BAFF furin cleavage site impairs B-cell homeostasis and antibody responses. Eur J Immunol. 2011;41(3):787-797.

31. Rochas C, Hillion S, Saraux A, et al. Transmembrane BAFF from rheumatoid synoviocytes requires interleukin- 6 to induce the expression of recombination-activating gene in B lymphocytes. Arthritis Rheum. 2009;60(5):1261-1271.

32. Chu VT, Enghard P, Riemekasten G, Berek C. In vitro and in vivo activation induces BAFF and APRIL expression in B cells. J Immunol. 2007;179(9):5947-5957.

33. Abu-Rish EY, Amrani Y, Browning MJ. Toll-like receptor 9 activation induces expression of membrane-bound B-cell activating factor (BAFF) on human B cells and leads to increased proliferation in response to both soluble and membrane-bound BAFF. Rheumatology (Oxford). 2013;52(7):1190-1201.

34. Kikly K, Manetta J, Smith H, Wierda D, Witcher D. Characterization of LY2127399, A neutralizing antibody for BAFF. Arthritis Rheum. 2009;60:S10.

35. Genovese MC, Bojin S, Biagini IM, et al. Tabalumab in rheumatoid arthritis patients with an inadequate response to methotrexate and naive to biologic therapy: a phase II, randomized, placebo-controlled trial. Arthritis Rheum. 2013;65(4):880-889.

36. Badr G, Borhis G, Lefevre EA, et al. BAFF enhances chemotaxis of primary human B cells: a particular synergy between BAFF and CXCL13 on memory B cells. Blood. 2008;111(5):2744-2754.

37. Wallace DJ, Stohl W, Furie RA, et al. A phase II, randomized, double-blind, placebo- controlled, dose-ranging study of belimumab in patients with active systemic lupus erythematosus. Arthritis Rheum. 2009;61(9):1168-1178.

38. van Vollenhoven RF, Kinnman N, Vincent E, Wax S, Bathon J. Atacicept in patients with rheumatoid arthritis and an inadequate response to methotrexate: results of a phase II, randomized, placebocontrolld trial. Arthritis Rheum. 2011;63(7):1782-1792.
Journal of Inflammation Research

\section{Publish your work in this journal}

The Journal of Inflammation Research is an international, peer-reviewed open-access journal that welcomes laboratory and clinical findings on the molecular basis, cell biology and pharmacology of inflammation including original research, reviews, symposium reports, hypothesis formation and commentaries on: acute/chronic inflammation; mediators of inflamma-

\section{Dovepress}

tion; cellular processes; molecular mechanisms; pharmacology and novel anti-inflammatory drugs; clinical conditions involving inflammation. The manuscript management system is completely online and includes a very quick and fair peer-review system. Visit http://www.dovepress.com/ testimonials.php to read real quotes from published authors. 